\title{
Renal safety of tenofovir containing antiretroviral regimen in a Singapore cohort
}

\author{
Arlene C Chua ${ }^{1 *}$, Ryan M Llorin ${ }^{1}$, Kelvin Lai ${ }^{2}$, Philippe Cavailler ${ }^{3}$ and Hwa Lin Law ${ }^{2}$
}

\begin{abstract}
Background: Tenofovirdisoproxilfumarate (TDF) is a nucleotide analogue widely recommended in international HIV treatment guidelines. The association of TDF and renal dysfunction has remained an area of interest.

Findings: We conducted a retrospective review of all patients on TDF from July 2007 to December 2009 in our institution and evaluated their renal function. Absolute change of creatinine clearance $\left(\mathrm{CL}_{\mathrm{Cr}}\right)$ using Cockroft-Gault equation from baseline was calculated at 6, 12, 18 and 24 months. Overall, 226 patients were included in the study. Ninety percent were male. The median age was 46 yrs old (23-82), median weight was $60 \mathrm{~kg}$ (IQR 53.75-68), median CD4 count was 127 cells $/ \mathrm{mm}^{3}$ (IQR 38-258) and median $\mathrm{CL}_{\mathrm{Cr}} 82.7 \mathrm{~mL} / \mathrm{min}$ (IQR 71.4-101.7) on initiation of TDF. The median decline of $\mathrm{CL}_{\mathrm{Cr}}$ from baseline was $-3.9 \mathrm{ml} / \mathrm{min}$ (IQR -12.3 to 7.6$)$, and $-3.6 \mathrm{ml} / \mathrm{min}$ (IQR -12.4 to 6.7) at $12(n=102), 24$ months $(n=75)$ respectively. Eighteen of 226 patients had a decline in renal function to $</=50 \mathrm{ml} / \mathrm{min}$. Majority of which had an improvement of $\mathrm{CL}_{\mathrm{Cr}_{r}}$ on follow up. Only $80 \%$ of patients ever received monitoring of renal function.

Conclusion: While we noted renal toxicity to be rare and transient among our cohort receiving TDF as part of their ARV regimen, these results reflect the short term renal effects of TDF given that ARV treatment is lifelong. Given that laboratory monitoring may be difficult to implement in many situations, future prospective studies looking into an evidence based algorithm for less frequent renal function monitoring than current guideline recommendations may be helpful.
\end{abstract}

Keywords: Tenofovir, Kidney, Antiretrovirals

\section{Background}

Tenofovirdisoproxilfumarate (TDF) is a nucleotide analogue widely recommended in international HIV treatment guidelines [1-3].

The association of TDF and renal dysfunction has remained an area of interest. In several clinical trials, TDF has been found to be safe with renal effects reported to be $1-3 \%$ with minimal differences from comparative non TDF arms [4-7]. However, several case reports and series show patients treated with TDF may present with acute renal failure, Fanconi syndrome and diabetes insipidus. These reports suggested that TDF could cause renal tubular injury and decline in creatinine clearance $\left(\mathrm{CL}_{\mathrm{Cr}}\right)$ [8-14]. A meta-analysis of prospective studies comparing TDF-containing with non-TDF

\footnotetext{
*Correspondence: Arlene_CHUA@ttsh.com.sg

'Department of Infectious Disease, Tan Tock Seng Hospital, 11 Jalan Tan

Tock Seng, Singapore 308433, Singapore

Full list of author information is available at the end of the article
}

containing ARV regimens showed that TDF use was associated with statistically significant loss of renal function and the clinical effect was modest [15].

The mechanism by which TDF causes renal toxicity is not well characterized and some studies suggest TDF induces mitochondrial toxicity [16]. Pharmacogenetics has been explored as one potential risk factor [17]. Goicoechea et al have reported treatment with TDF and a protease inhibitor (PI) to be associated with greater declines in renal function compared to TDF with nonnucleoside reverse transcriptase inhibitor (NNRTI) based therapy [18].

Current international guidelines [2] recommend monitoring renal function at ARV initiation or modification, 2-8 weeks post ARV initiation or modification and every 3-6 months thereafter and when clinically indicated. Dose reduction for TDF is recommended when the falls $\mathrm{CL}_{\mathrm{Cr}}$ below $50 \mathrm{ml} / \mathrm{min}$. 
We evaluated our practice of monitoring for renal function in our local cohort of HIV infected individuals receiving TDF in Singapore as well as investigated the changes of renal function over time. We specifically looked at the proportion of patients whose $\mathrm{CL}_{\mathrm{Cr}}$ fell below $50 \mathrm{ml} / \mathrm{min}$ requiring TDF dose adjustment after treatment and subsequent management of these cases.

\section{Methods}

We conducted a retrospective review of all patients who were started on an antiretroviral regimen containing TDF from July 2007 to December 2009 at the Communicable Disease Centre (CDC) at Tan Tock Seng Hospital (TTSH) in Singapore. Data was collected from review of medical records. Data on demographics, ARV, comorbidities and TDF dose were collected. Creatinine clearance $\left(\mathrm{CL}_{\mathrm{Cr}}\right)$ was calculated using the CockcroftGault equation.

Absolute change of $\mathrm{CL}_{\mathrm{Cr}}$ from baseline was calculated for each patient at $3,6,9,12,15,18,21$ and 24 months. Not all patients had the same frequency of serum creatinine monitoring because of physician differences in clinical management. When available, the $\mathrm{CL}_{\mathrm{Cr}}$ at follow up was calculated using the serum creatinine level closest to the period of evaluation (+/- 1 month). Evaluations were made on the associations of other variables with change in $\mathrm{CL}_{\mathrm{Cr}}$ including age, ethnicity, sex, diabetes, hypertension, hepatitis $\mathrm{B}$ coinfection, presence of chronic renal disease, CD4 cell count and NNRTI or PI based regimen.

Monitoring of serum creatinine, appropriateness of dose of TDF based on $\mathrm{CL}_{\mathrm{Cr}}$, and deaths among those who developed renal toxicity were evaluated.

We defined renal toxicity as a reduction of $\mathrm{CL}_{\mathrm{Cr}}$ to $</=50 \mathrm{ml} / \mathrm{min}$. It is recommended that the dosing interval of TDF be modified for patients with a $\mathrm{CL}_{\mathrm{Cr}}$ of $<50$. Bygrave et al published their experience with TDF defining renal toxicity by a reduction of $\mathrm{CL}_{\mathrm{Cr}}$ to $</=50 \mathrm{ml} / \mathrm{min}$ [19].

For the baseline characteristics we used medians and interquartile ranges (IQRs) for continuous variables while counts and proportions were used for categorical variables. The proportion of creatinine measurements performed at baseline and follow up were calculated. The proportion of patients who received an appropriate TDF dose based on baseline $\mathrm{CL}_{\mathrm{Cr}}$ was obtained. The median change of $\mathrm{CL}_{\mathrm{Cr}}$ from baseline was calculated at $3,6,9,12,15,18,21$ and 24 months. SPSS for Windows version 18.0 (SPSS Inc. Chicago, IL, USA) was used for all statistical analysis. All tests were two tailed. In all tests, a $\mathrm{p}$ value $<0.05$ was considered significant. We compared proportions using Fisher's exact test, means using ANOVA test and medians using Kruskal Wallis test.
This study was reviewed and approved by the Singapore National Health Care Group (NHG) Domain Specific Ethics Review Board Committee.

\section{Results}

From July 2007 to December 2009, there were a total of 226 patients who received an antiretroviral regimen containing TDF. Baseline demographic and clinical characteristics of patients receiving TDF are presented in Table 1.

A total of 16 patients had discontinuation of TDF. The reasons for stopping therapy included virological failure $(n=11)$, change of TDF to another NRTI due to patient

Table 1 Characteristics of patients who received Tenofovir, TTSH, Singapore, Jul. 2007 - Dec. 2009 ( $N=226)$

\begin{tabular}{|c|c|}
\hline & No (\%) of patients at TDF Initiation \\
\hline \multicolumn{2}{|l|}{ Sex } \\
\hline Male & $203(89.9)$ \\
\hline Female & $23(10.1)$ \\
\hline \multicolumn{2}{|l|}{ Ethnicity } \\
\hline Chinese & $176(78.6)$ \\
\hline Malay & $26(11.6)$ \\
\hline Indian & $10(4.5)$ \\
\hline Others & $12(5.3)$ \\
\hline \multicolumn{2}{|l|}{ Age } \\
\hline Median (range) & $46(23-82)$ \\
\hline \multicolumn{2}{|l|}{ Weight } \\
\hline Median (IQR) & $60.0[53.75-68.0]$ \\
\hline \multicolumn{2}{|l|}{ ART Regimen } \\
\hline NNRTI-based & $118(52.2)$ \\
\hline PI - based & $108(47.8)$ \\
\hline \multicolumn{2}{|l|}{ Co-morbidity } \\
\hline Hepatitis B co-infection & $63(27.9)$ \\
\hline Diabetes mellitus & $26(11.5)$ \\
\hline Hypertension & $23(10.2)$ \\
\hline Chronic renal disease & $2(0.9)$ \\
\hline \multicolumn{2}{|l|}{ CD4 cell count (cells $/ \mathrm{mm}^{3}$ ) } \\
\hline Median [IQR] & $127[38-258]$ \\
\hline \multicolumn{2}{|l|}{ Viral Load (Log 10 Copies/mL) } \\
\hline Median [IQR] & $4.9[4.2-5.5]$ \\
\hline \multicolumn{2}{|l|}{ Creatinine clearance (mL/min) } \\
\hline Median [IQR] & $82.7[71.4-101.7]$ \\
\hline$>/=90$ & $72(43.3)$ \\
\hline $60-89$ & $73(43.9)$ \\
\hline $30-59$ & $20(12.2)$ \\
\hline$<30$ & $1(0.6)$ \\
\hline
\end{tabular}


preference unrelated to TDF toxicity $(\mathrm{n}=3)$ and renal toxicity $(\mathrm{n}=2)$.

There were $166(73.5 \%)$ patients with a baseline creatinine measured at initiation of TDF and the median $\mathrm{CL}_{\mathrm{Cr}}$ was $82.7 \mathrm{~mL} / \mathrm{min}$ (IQR 71.4-101.7). Subsequent serum creatinine was obtained in 192 (84.9\%) patients within 6 months and 202 (89.4\%) within 12 months of starting TDF. The patients did not have the same frequency of serum creatinine measurements during the follow up period. Creatinine has been checked for only $53 \%(102 / 194)$ and $77 \%$ (75/98) for patients in follow up at 12 and 24 month respectively.

Of those patients who had a baseline serum creatinine at initiation, 12 patients had a calculated $\mathrm{CL}_{\mathrm{Cr}}$ of $<50 \mathrm{~mL} /$ min. Among these, 11/12 (92\%) had an incorrect TDF dose. The dose of TDF was adjusted for 5 patients on the following visit, and for 3 patients, repeat $\mathrm{CL}_{\mathrm{Cr}}$ was $>50 \mathrm{~mL} / \mathrm{min}$ requiring no further TDF dose adjustment.

The median decline of $\mathrm{CL}_{\mathrm{Cr}}$ from baseline of $\mathrm{CL}_{\mathrm{Cr}}$ was $-3.9 \mathrm{ml} / \mathrm{min}$ (IQR -12.3 to 7.6 ) and $-3.6 \mathrm{ml} /$ min (IQR -12.4 to 6.7$)$ at $12(\mathrm{n}=102)$ and 24 months $(\mathrm{n}=75)$ respectively (Figure 1$)$. There was no statistical association between the trend in $\mathrm{CL}_{\mathrm{Cr}}$ and the CD4 count,, age, NNRTI or PI based ARV regimen, or the presence of an existing co-morbidity (Hepatitis $B$ coinfection, hypertension or diabetes).

There were 18 patients who had a baseline $\mathrm{CL}_{\mathrm{Cr}}$ of $>50 \mathrm{~mL} / \mathrm{min}$ who developed toxicity on follow up with a subsequent $\mathrm{CL}_{\mathrm{Cr}}$ of $\angle /=50 \mathrm{~mL} / \mathrm{min}$. The onset of $\mathrm{CL}_{\mathrm{Cr}}$ decline to $</=50 \mathrm{~mL} / \mathrm{min}$ in $10 / 18$ patients was within 6 months of TDF initiation. The $\mathrm{CL}_{\mathrm{Cr}}$ decline was $>25 \%$ for 16 patients and $>50 \%$ for 2 patients. Only 1 patient developed severe renal impairment $\left(\mathrm{CL}_{\mathrm{Cr}}<30 \mathrm{ml} / \mathrm{min}\right)$.
There were 3/18 that died of which none were attributed to TDF toxicity. Of the 15 remaining patients, 2 were switched to a non TDF containing regimen and 13 patients remained on TDF despite renal impairment. The $\mathrm{CL}_{\mathrm{Cr}}$ improved without dose adjustment in the majority of these patients (10 of 13). The remaining 3/10 patients had TDF dose adjustment to $300 \mathrm{mg}$ once every other day based on reduced $\mathrm{CL}_{\mathrm{Cr}}$.

We compared these 18 patients with those who did not have any renal toxicity and found no differences in terms of their baseline CD4 count, age, gender, presence of hypertension, diabetes, or hepatitis $\mathrm{B}$ status. There were $12 / 18$ patients who were on a PI based regimen. There was a tendency for reduction of $\mathrm{Cl}_{\mathrm{Cr}}$ among those who had a PI based regimen ( $\mathrm{p}=0.38$, at 3 months) however this was not significant.

\section{Discussion}

Our study showed that in an Asian HIV population, majority of whom had a low CD4 count but relatively normal $\mathrm{CL}_{\mathrm{Cr}}$ the proportion of patients who develop renal toxicity is low $(2.2 \% ; 5 / 226)$. While use of protease inhibitor has been associated with greater decline in renal function [18], we are unable to show any significant difference which may be attributed to the small number in our study.

In our clinical practice setting around $80 \%$ of patients on TDF ever received monitoring of renal function.

The data on TDF related renal toxicity in an Asian HIV cohort is limited. A recent observational Japanese cohort study showed that the incidence of renal dysfunction in low body weight patients treated with tenofovir was twice as high compared to those treated with

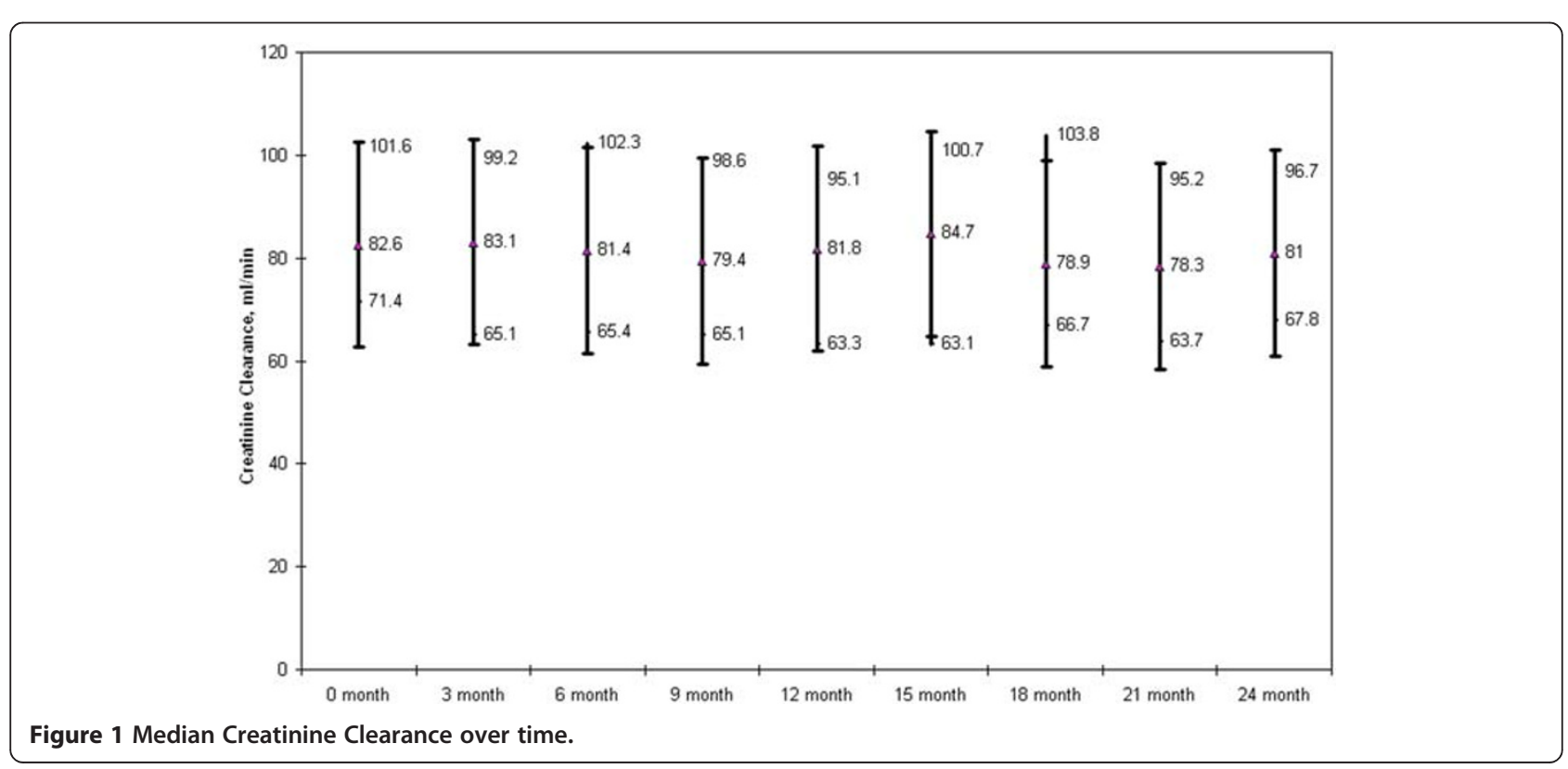


abacavir [20]. A study looking at the incidence and predictor of TDF associated renal toxicity in Thai patients is currently ongoing [21].

One limitation of our study is that we calculated $\mathrm{CL}_{\mathrm{Cr}}$ using the Cockroft-Gault equation. Studies have shown that the abbreviated MDRD equation performs better than the Cockroft Gault equation for non Caucasian ethnic groups [22-24].

Other limitations of our study are that we did not have a non TDF comparator group. Our follow up period is limited to up to 24 months which only 98 out of 226 patients have achieved. It would be important to see $\mathrm{CL}_{\mathrm{Cr}}$ trend over longer periods as other studies have shown decline in renal function long term $[25,26]$.

Limitations of using retrospective data obtained from medical records include incomplete data, difficulty in verifying documented information, and variability in the quality of documentation among physicians. Moreover, not all patients had creatinine measured during the designated follow up period due to physician variability in clinical practice. Our study cohort includes both ARV naïve and experienced patients which makes our results difficult to compare with other studies.

Our study showed that renal toxicity was uncommon and transient which confirms previous studies on TDF. Given that ARV therapy is lifelong, the results of our study reflect the short term renal effects of TDF. Current international guidelines recommend monitoring renal function at ARV initiation or modification, 2-8 weeks and every 3-6 months thereafter [2]. Given that laboratory monitoring may be difficult to implement in many situations, future prospective studies looking into an evidence based algorithm for less frequent renal function monitoring than current guideline recommendations may be helpful.

\section{Competing interests}

All authors declare that they have no competing interests.

\section{Author's contributions}

AC wrote the manuscript, designed the study and analyzed the data. RL participated in the collection of data. PC participated in the collection of data and analyzed the data. KL participated in the collection of data. $\mathrm{HL}$ participated in the design of the study. All authors have read and approved the final manuscript.

\footnotetext{
Acknowledgements

The study was approved by the Domain Specific Review Board of the National Healthcare Group in Singapore.

The authors would like to acknowledge Muhamad Alif Ibrahim from the Department of Clinical Epidemiology, Tan Tock Seng Hospital, Singapore for his help in preparing the manuscript for publication.

\section{Author details}

'Department of Infectious Disease, Tan Tock Seng Hospital, 11 Jalan Tan Tock Seng, Singapore 308433, Singapore. ${ }^{2}$ Department of Pharmacy, Tan Tock Seng Hospital, 11 Jalan, Tan Tock Seng, Singapore 308433, Singapore. ${ }^{3}$ Department of Epidemiology Research, Regional Emerging Diseases Intervention Centre, 10 Biopolis Road \#02-01, Singapore 138670, Singapore.
}

Received: 10 January 2012 Accepted: 15 May 2012

Published: 15 June 2012

\section{References}

1. Bucher $\mathrm{HC}$, Wolbers M, Porter K: Guidelines for antiretroviral treatment of HIV from the International AIDS Society-USA Panel. JAMA 2010, 304 (17):1897-1898.

2. Panel on Antiretroviral Guidelines for Adults and Adolescents: Guidelines for the use of antiretroviral agents in HIV-1-infected adults and adolescents. Editor: Department of Health and Human Services; 2011.

3. World Health Organization: HIV/AIDS Department, Antiretroviral Therapy For HIV Infection in Adults and Adolescents, Recommendations for a Public Health Approach. Editor: WHO; 2010.

4. Arribas JR, et al: Tenofovir disoproxil fumarate, emtricitabine, and efavirenz compared with zidovudine/lamivudine and efavirenz in treatment-naive patients: 144-week analysis. J Acquir Immune Defic Syndr 2008, 47(1):74-78.

5. Gallant JE, et al: Efficacy and safety of tenofovir DF vs stavudine in combination therapy in antiretroviral-naive patients: a 3-year randomized trial. JAMA 2004, 292(2):191-201.

6. Izzedine $\mathrm{H}$, et al: Long-term renal safety of tenofovir disoproxil fumarate in antiretroviral-naive HIV-1-infected patients. Data from a double-blind randomized active-controlled multicentre study. Nephrol Dial Transplant 2005, 20(4):743-746.

7. Padilla $\mathrm{S}$, et al: Low frequency of renal function impairment during oneyear of therapy with tenofovir-containing regimens in the real-world: a case-control study. AIDS Patient Care STDS 2005, 19(7):421-424.

8. Barrios A, et al: Tenofovir-related nephrotoxicity in HIV-infected patients. AIDS 2004, 18(6):960-963.

9. Irizarry-Alvarado $\mathrm{JM}$, et al: Proximal tubular dysfunction associated with tenofovir and didanosine causing Fanconi syndrome and diabetes insipidus: a report of 3 cases. AIDS Read 2009, 19(3):114-121.

10. Izzedine $H$, et al: Renal safety of tenofovir in HIV treatment-experienced patients. AIDS 2004, 18(7):1074-1076.

11. Peyriere $\mathrm{H}$, et al: Renal tubular dysfunction associated with tenofovir therapy: report of 7 cases. J Acquir Immune Defic Syndr 2004, 35(3):269-273.

12. Quimby D, Brito MO: Fanconi syndrome associated with use of tenofovir in HIV-infected patients: a case report and review of the literature. AIDS Read 2005, 15(7):357-364

13. Verhelst $D$, et al: Fanconi syndrome and renal failure induced by tenofovir: a first case report. Am J Kidney Dis 2002, 40(6):1331-1333.

14. Williams J, Chadwick DR: Tenofovir-induced renal tubular dysfunction presenting with hypocalcaemia. J Infect 2006, 52(4):e107-e108.

15. Cooper RD, et al: Systematic review and meta-analysis: renal safety of tenofovir disoproxil fumarate in HIV-infected patients. Clin Infect Dis 2010, 51(5):496-505.

16. Kohler JJ, et al: Tenofovir renal toxicity targets mitochondria of renal proximal tubules. Lab Invest 2009, 89(5):513-519.

17. Rodriguez-Novoa S, et al: Predictors of kidney tubular dysfunction in HIVinfected patients treated with tenofovir: a pharmacogenetic study. Clin Infect Dis 2009, 48(11):e108-e116.

18. Goicoechea M, et al: Greater tenofovir-associated renal function decline with protease inhibitor-based versus nonnucleoside reversetranscriptase inhibitor-based therapy. J Infect Dis 2008, 197(1):102-108.

19. Bygrave $\mathrm{H}$, et al: Renal safety of a tenofovir-containing first line regimen: experience from an antiretroviral cohort in rural Lesotho. PLOS One 2011, 6(3):e17609.

20. Nishijima T, et al: Renal function declines more in tenofovir- than abacavir-based antiretroviral therapy in low-body weight treatmentnaive patients with HIV infection. PLoS One 2012, 7(1):e29977.

21. The HIV Netherlands Australia Thailand Research, Collaboration: Tenofovir Renal Toxicity and Glomerular Filtration Rate (GFR) Validation: ; 2011. Available from: http://clinicaltrials.gov/ct2/show/study/NCT01138241.

22. Levey AS, et al: A more accurate method to estimate glomerular filtration rate from serum creatinine: a new prediction equation. Modification of Diet in Renal Disease Study Group. Ann Intern Med 1999, 130(6):461-470.

23. Levey AS, et al: Using standardized serum creatinine values in the modification of diet in renal disease study equation for estimating glomerular filtration rate. Ann Intern Med 2006, 145(4):247-254. 
24. Zuo L, et al: Application of GFR-Estimating Equations in Chinese Patients With Chronic Kidney Disease. Am J Kidney Dis 2005, 45(3):463-472.

25. Dauchy FA, et al: Increased risk of abnormal proximal renal tubular function with HIV infection and antiretroviral therapy. Kidney Int 2011, 80 (3):302-309.

26. Wever $K$, van Agtmael MA, Carr A: Incomplete reversibility of tenofovirrelated renal toxicity in HIV-infected men. J Acquir Immune Defic Syndr 2010, 55(1):78-81.

doi:10.1186/1742-6405-9-19

Cite this article as: Chua et al:: Renal safety of tenofovir containing antiretroviral regimen in a Singapore cohort. AIDS Research and Therapy 2012 9:19.

\section{Submit your next manuscript to BioMed Central and take full advantage of:}

- Convenient online submission

- Thorough peer review

- No space constraints or color figure charges

- Immediate publication on acceptance

- Inclusion in PubMed, CAS, Scopus and Google Scholar

- Research which is freely available for redistribution 